\title{
Two-stage SPIT detection scheme with betweenness centrality and social trust
}

\author{
Miho Kurata ${ }^{\text {a) }}$, Kentaroh Toyoda ${ }^{\text {a)}}$, and Iwao Sasase \\ Dept. of Information and Computer Science, Keio University, \\ 3-14-1 Hiyoshi, Kohoku, Yokohama, Kanagawa 223-8522, Japan \\ a)toyoda+kurata@sasase.ics.keio.ac.jp
}

\begin{abstract}
Detecting SPIT (Spam over Internet Telephony) is an urgent demand in voice communication services. In this paper, we propose a twostage SPIT detection scheme using BC (Betweenness Centrality) and social trust to decrease misdetection of a call from low-frequent users as SPIT. BC indicates user's centrality in the entire network and the $\mathrm{BC}$ against legitimate users gradually increases with time even if users seldom call. We first use $\mathrm{BC}$ to identify a call request from a low-frequent user then judge the call legitimacy by using social trust. By the computer simulation, we show that our scheme improves the detection accuracy.
\end{abstract}

Keywords: SPIT detection, VoIP, security, social trust Classification: Internet

\section{References}

[1] A. D. Keromytis, "A comprehensive survey of voice over IP security research," IEEE Comm. Surv. and Tutor., vol. 14, no. 2, pp. 514-537, 2012. DOI:10.1109/ SURV.2011.031611.00112

[2] J. Seedorf, N. D'Heureuse, S. Niccolini, and M. Cornolti, "Detecting trustworthy real-time communications using a Web-of-Trust," IEEE GLOBECOM, pp. 1-8, 2009. DOI:10.1109/GLOCOM.2009.5425529

[3] T. Kusumoto, E. Y. Chen, and M. Itoh, "Using call patterns to detect unwanted communication callers," IEEE/IPSJ International Symposium on Applications and the Internet (SAINT), pp. 64-70, 2009. DOI:10.1109/SAINT.2009.19

[4] M. A. Azad and R. Morla, "Caller-REP: Detecting unwanted calls with caller social strength,” Comput. Secur., vol. 39, Part B, pp. 219-236, 2013. DOI:10. 1016/j.cose.2013.07.006

[5] N. Chaisamran, T. Okuda, and S. Yamaguchi, "Trust-based VoIP spam detection based on calling behaviors and human relationships," J. Inf. Process., vol. 21, no. 2, pp. 188-197, 2013. DOI:10.2197/ipsjjip.21.188

[6] U. Brandes, "A faster algorithm for betweenness centrality," J. Math. Sociol., vol. 25, no. 2, pp. 163-177, 2001. DOI:10.1080/0022250X.2001.9990249

\section{Introduction}

Various voice communication services are getting popular with the growing smartphone market. However, it is reported that malicious users or companies 
may abuse them for advertisement or fraud, which is called SPIT (Spam over Internet Telephony) [1]. Hence, detecting SPIT calls or spammers in voice communication services is an urgent demand for the service providers. In particular, a social trust-based approach is receiving much attention due to growing SNS-based voice communication services. A social trust-based approach judges the legitimacy of a call with a trust value calculated from caller-callee relationships $[2,3,4,5]$. We especially pay attention to the scheme [5] since it can correctly classify unknown users. This scheme uses the call duration as the trust value and the longer a user calls to a callee, the higher trust value the callee gives to the caller. However we notice that the scheme [5] raises false alarms for low-frequent legitimate users as time goes on. That is, the trust value of low-frequent users gradually decreases since they seldom receive calls.

In this paper, we propose a two-stage SPIT detection scheme with BC (Betweenness Centrality) and social trust. We use $\mathrm{BC}$ to allow a call request from a low-frequent legitimate user at the first stage. And then we judge the legitimacy of a call by using the conventional scheme [5]. BC indicates how much a user is gone through shortest paths between paths among other users. The intuition behind utilizing $\mathrm{BC}$ is that spammers call towards users while they seldom receive calls and thus spammers tend to be 'isolated' at the edge of the entire network. On the other hand, the value of $\mathrm{BC}$ against legitimate users gradually increases with time even if users seldom call. By the computer simulation, we show the legitimacy of introducing $\mathrm{BC}$ and also clarify that our scheme improves the detection accuracy.

\section{System model}

We define a voice-based spammer as the attacker model and the aims of spammers are advertisement, voice phishing, and illegal sales. Spammers call towards randomly chosen users but they seldom receive calls from others. The call frequency is higher than that of legitimate users. Since the contents of SPIT seems to irritate ordinary users, the call duration tends to be much shorter than that of legitimate users.

We assume that a SPIT detection system is deployed in a voice communication service provider and its task is to judge whether a call request should be established or not when receiving a call request from a user. We assume that as many as $N_{\text {user }}$ users (including both legitimate users and spammers) in the service provider and the system can access to users' CDR (Call Detail Records) and buddy lists (friend lists) for the inspection.

\section{Conventional scheme}

Chaisamran et al. propose a voice-based SPIT detection scheme with a social trust [5]. This scheme always allows a call from a user in the callee's buddy list. Otherwise, i.e., if a call is from an unknown user, the system judges the legitimacy of call with an inferred trust value calculated from trust values of other users. Since multiple paths between an unknown caller $u$ and a callee $v$ may exist, the system chooses the maximum inferred trust $T_{u \rightarrow v}$ as shown in Eq. (1). 


$$
T_{u \rightarrow v}=\max _{p \in P_{u \rightarrow v}}\left(T_{u \rightarrow v}^{\text {path }_{p}}\right),
$$

where $P_{u \rightarrow v}$ denotes a set of paths between $u$ and $v$ and $T_{u \rightarrow v}^{p a t h_{p}}$ indicates an inferred trust value calculated between users in a path $p$ and is represented as Eq. (2).

$$
T_{u \rightarrow v}^{\text {path }_{p}}=\prod_{i \in p} T_{i(t)} .
$$

In order to make a trust value reliable, each user is assigned a trust value from his/ her friend depending on the cumulative call duration. This will give a low trust value for spammers since they seldom receive calls. More specifically, a user $i$ in the path $p$ has its own trust value at time $t$ as Eq. (3).

$$
T_{i(t)}=\alpha R_{i(t)}+(1-\alpha) T_{i(t-1)},
$$

where $\alpha$ denotes a weight variable $(\alpha \in[0,1])$ and a raw trust value $R_{i(t)}$ at time $t$ is represented as Eq. (4).

$$
R_{i(t)}=\frac{C_{v(t)}}{\left(\prod_{j=1}^{n} C_{j(t)}\right)^{\frac{1}{n}}},
$$

where $C_{j(t)}$ denotes the cumulative call duration that a user $j$ calls to user $v$ and $n$ denotes the number of $v$ 's friends, respectively.

Finally, the system compares the inferred trust value $T_{u \rightarrow v}$ and the pre-defined threshold $T_{t h}$. If $T_{u \rightarrow v}>T_{t h}$, the system establishes a call request from a user $u$ to $v$. Otherwise, it rejects a call request.

\subsection{Shortcomings of the conventional scheme}

We argue that the scheme [5] raises false alarms for low-frequent legitimate users as time goes on. That is, the trust value of low-frequent users gradually decreases since they seldom receive calls.

\section{Proposed scheme}

Here, we propose a two-stage SPIT detection scheme with BC and social trust in order for the system to correctly identify a call request from low-frequent legitimate users as a legitimate one. We use $\mathrm{BC}$ as a feature to allow a call from low-frequent users at the first stage. After that, we judge the legitimacy of a call by using the social trust-based approach [5].

\subsection{Introduction of BC}

In graph theory, BC indicates a user's centrality in the social network [6]. Formally, $\mathrm{BC}$ is defined as the ratio of the number of shortest paths from all users to all others that pass through that user. Let $\sigma_{s t}$ denote the number of shortest paths from $s \in U$ to $t \in U$, where $U$ denotes a set of users in the entire network. Let $\sigma_{s t}(u)$ denote the number of shortest paths from $s \in U$ to $t \in U$ that pass through $u \in U$. By using $\sigma_{s t}$ and $\sigma_{s t}(u), \mathrm{BC}(u)$, which is the $\mathrm{BC}$ of a user $u$, can be represented as Eq. (5).

$$
\mathrm{BC}(u)=\sum_{s \neq u \neq t \in U} \frac{\sigma_{s t}(u)}{\sigma_{s t}} .
$$




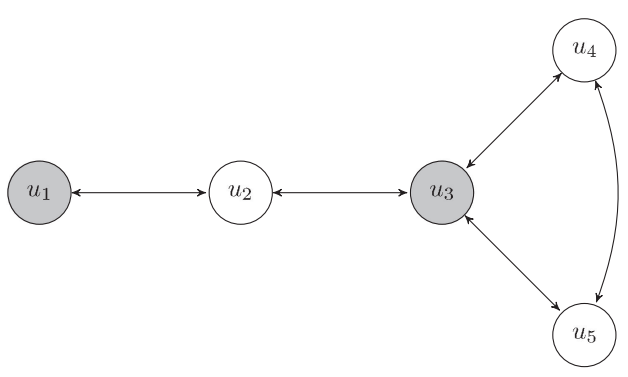

Fig. 1. Toy example of SNS that consists of five users.

The above description is rigid and difficult to understand why Eq. (5) indicates the centrality. Hence we calculate BC with an SNS that consists of five users with Fig. 1. In this example, we calculate two users' $\mathrm{BC}$, which are $u_{1}$ and $u_{3}$ and they are represented as $\mathrm{BC}\left(u_{1}\right)=\frac{\sigma_{2,3}\left(u_{1}\right)}{\sigma_{2,3}}+\frac{\sigma_{2,4}\left(u_{1}\right)}{\sigma_{2,4}}+\frac{\sigma_{2,5}\left(u_{1}\right)}{\sigma_{2,5}}+\frac{\sigma_{3,4}\left(u_{1}\right)}{\sigma_{3,4}}+\frac{\sigma_{3,5}\left(u_{1}\right)}{\sigma_{3,5}}+\frac{\sigma_{4,5}\left(u_{1}\right)}{\sigma_{4,5}}=$ $\frac{0}{1}+\frac{0}{1}+\frac{0}{1}+\frac{0}{1}+\frac{0}{1}+\frac{0}{1}=0$ and $\mathrm{BC}\left(u_{3}\right)=\frac{0}{1}+\frac{1}{1}+\frac{1}{1}+\frac{1}{1}+\frac{1}{1}+\frac{0}{1}=4$. Therefore, $\mathrm{BC}\left(u_{3}\right)>\mathrm{BC}\left(u_{1}\right)$ and thus the user $u_{3}$ is located more central than the user $u_{1}$. This matches the fact that the user $u_{1}$ is located at the edge of the entire network while the user $u_{3}$ is located in the center of the network in Fig. 1.

We argue that $\mathrm{BC}$ for spammers does not increase. Since spammers call but seldom receive calls, they tend to be 'isolated' at the edge of the entire network and hardly go through the shortest paths between users. Hence the numerator of Eq. (5) for spammers does not increase well. On the other hand, BC for legitimate users gradually increases with time even if users seldom call. This is because legitimate users gradually make connection with legitimate users and the number of paths that go through legitimate users increases. Therefore, the numerator in Eq. (5) for legitimate users gradually increases. Spammers may collude each other to increase the value of their BC, which is so-called Sybil attack. Although they can "locally" increase their BC, it is difficult to "globally" increase it. Hence spammers should account for large part of entire user to succeed the Sybil attack. In reality, spammers would be less compared to the legitimate users and thus BC for spammers is still small even if they collude.

\subsection{Algorithm}

When receiving a call establish request from a caller $u$, the server first checks whether the caller $u$ is in the callee's buddy list. If the caller is in callee's buddy list, they are assumed to be friends and thus the system establishes a call request. Otherwise, the system proceeds to the first detection stage that checks whether his/ her $\mathrm{BC}(u)$ is bigger than a pre-defined threshold $\mathrm{BC}_{t h}$. If $\mathrm{BC}(u)>\mathrm{BC}_{t h}$, the system judges that the call is legitimate and establishes the call. Otherwise, the system calculates the inferred trust value $T_{u \rightarrow v}$ by using Eqs. (1)-(4) and checks whether the caller's inferred social trust from the callee $v T_{u \rightarrow v}$ is bigger than a pre-defined threshold $T_{t h}$. If $T_{u \rightarrow v}>T_{t h}$, the system judges that the call is legitimate and establishes a call. Otherwise, the system rejects the call establishment request. 


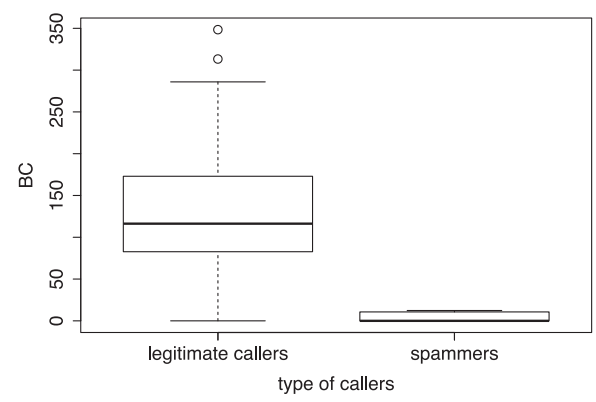

(a) BC versus the type of callers.

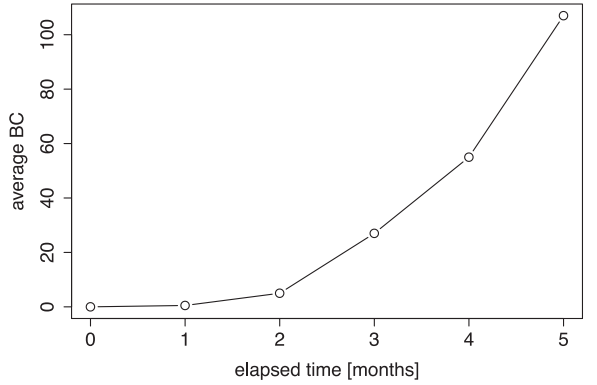

(b) $\mathrm{BC}$ versus elapsed times for newcommers.

Fig. 2. Characteristics of $\mathrm{BC}$.

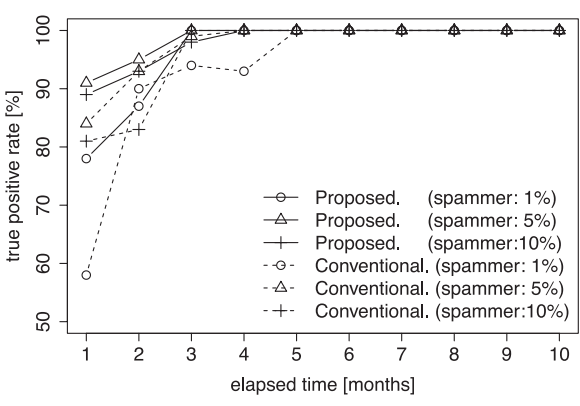

(a) True positive rate versus elapsed time.

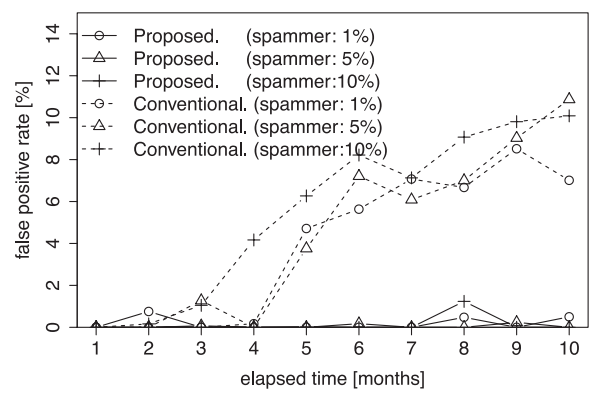

(b) False positive rate versus elapsed time.

Fig. 3. Detection accuracy.

\section{Simulation results}

We evaluate the characteristics of $\mathrm{BC}$ and the detection accuracy by the computer simulation. We use the simulation parameters specified in [5] and set the other undefined parameters as $N_{\text {user }}=1,000$ and $\mathrm{BC}_{t h}=50$.

\subsection{Characteristics of BC}

Fig. 2(a) shows the boxplot of an average $B C$ values against legitimate callers and spammers when five months passed. As we can see from Fig. 2(a), BC for spammers is concentrated around 0 while $\mathrm{BC}$ for legitimate callers mostly ranges from 50 to 200. Fig. 2(b) shows the average BC for legitimate users with time. As we can see from Fig. 2(b), the BC for legitimate users gradually increases with time.

\subsection{Detection accuracy}

Fig. 3(a) and Fig. 3(b) show the true positive rate and false positive rate versus elapsed time. The true positive rate denotes the ratio of correctly identified calls from spammers while the false positive rate is the ratio of mistakenly identified calls from legitimate users, respectively. We first discuss the true positive rate. From Fig. 3(a), we confirm that our scheme does not degrade the true positive rate. We then discuss the false positive rate. In Fig. 3(b), the false positive rate against the conventional scheme is getting worse with time. On the other hand, the false positive rate of the proposed scheme is within $2 \%$ and does not degrade with time. 
The false positive rate seems to be irrespective of the ratio of spammers. This is because the scheme judges whether each 'call request' (not 'caller') is legitimate or not. From this result, we can say that the false positive rate can be remedied by using $\mathrm{BC}$.

\section{Conclusion}

We have pointed out that calls from low-frequent users are gradually identified as SPIT in the conventional scheme. To remedy this issue, we have proposed a twostage SPIT detection scheme with BC and social trust. By the computer simulation, it is shown that our scheme achieves low false positive rate $(<2 \%)$ without lowering true positive rate.

\section{Acknowledgments}

This work is partly supported by the Grant in Aid for Scientific Research (No. 26420369) from Ministry of Education, Sport, Science and Technology, Japan. 